TRANSACTIONS OF THE

AMERICAN MATHEMATICAL SOCIETY

Volume 350, Number 10, October 1998, Pages 3967-3987

S $0002-9947(98) 02033-9$

\title{
ON THE CONNECTION \\ BETWEEN THE EXISTENCE OF ZEROS \\ AND THE ASYMPTOTIC BEHAVIOR OF RESOLVENTS \\ OF MAXIMAL MONOTONE OPERATORS \\ IN REFLEXIVE BANACH SPACES
}

\author{
ATHANASSIOS G. KARTSATOS
}

\begin{abstract}
A more systematic approach is introduced in the theory of zeros of maximal monotone operators $T: X \supset D(T) \rightarrow 2^{X^{*}}$, where $X$ is a real Banach space. A basic pair of necessary and sufficient boundary conditions is given for the existence of a zero of such an operator $T$. These conditions are then shown to be equivalent to a certain asymptotic behavior of the resolvents or the Yosida resolvents of $T$. Furthermore, several interesting corollaries are given, and the extendability of the necessary and sufficient conditions to the existence of zeros of locally defined, demicontinuous, monotone mappings is demonstrated. A result of Guan, about a pathwise connected set lying in the range of a monotone operator, is improved by including non-convex domains. A partial answer to Nirenberg's problem is also given. Namely, it is shown that a continuous, expansive mapping $T$ on a real Hilbert space $H$ is surjective if there exists a constant $\alpha \in(0,1)$ such that $\langle T x-T y, x-y\rangle \geq-\alpha\|x-y\|^{2}, x, y \in H$. The methods for these results do not involve explicit use of any degree theory.
\end{abstract}

\section{Introduction-Preliminaries}

In what follows, the symbol $X$ stands for a real Banach space with norm $\|\cdot\|$ and (normalized) duality mapping $J$. The symbols $\left\langle x^{*}, x\right\rangle$ and $\left\langle x, x^{*}\right\rangle$ denote the value of the functional $x^{*} \in X^{*}$ at $x \in X$. We denote by $D(T), R(T)$ and $G(T)$ the effective domain, the range and the graph of a mapping $T: X \rightarrow 2^{X^{*}}$, respectively. We have $D(T)=\{x \in X: T x \neq \emptyset\}$ and $G(T)=\left\{\left(x, x^{*}\right): x \in D(T), x^{*} \in T x\right\}$.

Unless otherwise stated, we shall assume that $X$ is a reflexive Banach space. We shall also assume that $X$ and $X^{*}$ have been renormed so that they are locally uniformly convex. This implies that the duality mapping $J$ is single-valued, strictly monotone (see definition below) and bicontinuous.

In what follows, "continuous" means "strongly continuous" and the symbol " $\rightarrow$ " ("- ") means strong (weak) convergence. The symbol $R\left(R_{+}\right)$stands for the set $(-\infty, \infty)([0, \infty))$ and the symbols $\partial D, \operatorname{int} D, \bar{D}$ denote the strong boundary, interior and closure of the set $D$, respectively. An operator $T: X \supset D(T) \rightarrow Y$, with $Y$ another Banach space, is "bounded" if it maps bounded subsets of $D(T)$

Received by the editors March 6, 1995 and, in revised form, November 7, 1996.

1991 Mathematics Subject Classification. Primary 47H17; Secondary 47H05, 47H10.

Key words and phrases. Maximal monotone operator, resolvent, Yosida resolvent, demicontinuous monotone operator, existence of zeros, Nirenberg's problem. 
onto bounded sets. It is "compact" if it is continuous and maps bounded subsets of $D(T)$ onto relatively compact sets. It is called "demicontinuous" if it is continuous on $D(T)$ from the strong topology to the weak topology. An operator $T: X \supset D(T) \rightarrow 2^{X^{*}}$ is "monotone" if

$$
\left\langle u^{*}-v^{*}, x-y\right\rangle \geq 0 \text { for every } x, y \in D(T) \text { and every } u^{*} \in T x, v^{*} \in T y .
$$

A monotone operator $T$ is "strongly monotone" if 0 in the right-hand side of $(*)$ is replaced by $\alpha\|x-y\|^{2}$, where $\alpha>0$ is a fixed constant. A monotone operator is called "strictly monotone" if equality in $(*)$ implies $x=y$. A monotone operator $T$ is called "maximal monotone" if its graph $G(T)$ is a maximal monotone subset of $X \times X^{*}$ when $X \times X^{*}$ is partially ordered by inclusion. A monotone operator $T$ is maximal monotone if and only if $R(T+\lambda J)=X^{*}$ for every $\lambda>0$.

Given a maximal monotone operator $T$ and $x \in X$, the equation

$$
J\left(x_{\lambda}-x\right)+\lambda x_{\lambda}^{*}=0,\left(x_{\lambda}, x_{\lambda}^{*}\right) \in G(T),
$$

has a unique solution $\left(x_{\lambda}, x_{\lambda}^{*}\right) \in G(T)$ for every $\lambda>0$. We set

$$
J_{\lambda} x \equiv x_{\lambda}, T_{\lambda} x \equiv x_{\lambda}^{*}, x \in X .
$$

It follows easily that, for all $x \in X$, we have $J_{\lambda} x=\left(I+\lambda J^{-1} T\right)^{-1} x$ and $T_{\lambda} x=$ $(1 / \lambda) J\left(x-J_{\lambda} x\right)$. Also, $T_{\lambda} x \in T J_{\lambda} x, x \in X$. We also know that $T_{\lambda}$ is a maximal monotone operator, while both $J_{\lambda}$ (which we call the "Yosida resolvent" of $T$ ) and $T_{\lambda}$ are demicontinuous and bounded. The demicontinuity of the operator $J_{\lambda}$ can also be found in the proof of Proposition 1.1 of Barbu [3]. These operators, $J_{\lambda}, T_{\lambda}$ were originally defined in the paper [4] by Brézis, Crandall and Pazy, where their fundamental properties were also obtained. They can also be found in the books of Barbu [3], Pascali and Sburlan [35] and Zeidler [40].

In this paper we give several necessary and sufficient conditions for the existence of a zero of a maximal monotone operator $T: X \supset D(T) \rightarrow 2^{X^{*}}$. Proposition 1 contains two such boundary conditions and extends to the present setting results from Reich and Torrejón [37] and Morales [30]. It is also related to various results involving ranges of maximal monotone and regular operators (cf. Pascali and Sburlan [35, 148-150]). The first corollary to this proposition improves a recent result of Yang [39]. Another corollary, Corollary 4, extends Altman's well-known fixed point theorem to the existence of zeros of maximal monotone operators. Two more results, Theorem 1 and Theorem 2, give necessary and sufficient conditions for the existence of zeros of maximal monotone operators in terms of resolvents and Yosida resolvents, respectively. Theorem 3 provides approximations of zeros of such operators. More precisely, the zeros of maximal monotone operators are obtained as limits of time-dependent resolvents when $t \rightarrow \infty$.

Section 3 is devoted to demicontinuous monotone operators defined on open, bounded subsets $G$ of $X$. In particular, Theorem 4 establishes a new necessary and sufficient condition for the existence of a zero of such an operator $T$, while Proposition 2 improves a result of Guan [10] involving conditions under which a pathwise connected set $M$ lies in the range of $T$. A partial answer to Nirenberg's problem is given in Section 4. We do not assume that $\operatorname{int} R(T) \neq \emptyset$, but we do assume a monotonicity-type condition on the relevant expansive operator. We have also shown that the solvability of Nirenberg's problem, in the affirmative, is equivalent to the maximal monotonicity of a related operator. 
Section 5 contains a discussion of our results as well as three open problems, the solution of which would lead to the further development of the relevant theories involving accretive and monotone operators.

For relevant results, for maximal monotone and $m$-accretive operators, we cite [3]-[40]. For certain applications to control theory we cite [16] and [28]. We need the following lemma which can be found in [4].

Lemma A. Let $T: X \supset D(T) \rightarrow 2^{X^{*}}$ be maximal monotone. Assume that $\left\{x_{n}\right\} \subset$ $D(T), x_{n}^{*} \in T x_{n}$ with $x_{n} \rightarrow x, x_{n}^{*} \rightarrow x^{*}$, and

$$
\limsup _{n \rightarrow \infty}\left\langle x_{n}^{*}-x^{*}, x_{n}-x\right\rangle \leq 0,
$$

or

$$
\limsup _{n, m \rightarrow \infty}\left\langle x_{n}^{*}-x_{m}^{*}, x_{n}-x_{m}\right\rangle \leq 0 .
$$

Then $\left(x, x^{*}\right) \in G(T)$ and $\left\langle x_{n}^{*}, x_{n}\right\rangle \rightarrow\left\langle x^{*}, x\right\rangle$. In particular, if $x_{n}^{*}=J x_{n}$, then $x^{*}=J x,\left\|x_{n}\right\|^{2}=\left\langle J x_{n}, x_{n}\right\rangle \rightarrow\langle J x, x\rangle=\|x\|^{2}$ and $x_{n} \rightarrow x$. $[5]$.

The next lemma can be found in the proof of Theorem 7.6 in Browder's book

Lemma B. Let $x \in X$ and $\left\{x_{n}\right\} \subset X$ be such that

$$
\lim _{n \rightarrow \infty}\left\langle J x_{n}-J x, x_{n}-x\right\rangle=0 .
$$

Then $x_{n} \rightarrow x$ as $n \rightarrow \infty$.

Lemma C. Let $\left\{s_{n}\right\}$ be a nonincreasing sequence of positive numbers, with $s_{n} \rightarrow 0$ as $n \rightarrow \infty$, and $\left\{x_{n}\right\} \subset X$. Assume that for all $m, n$ we have

$$
\left\langle s_{n} J x_{n}-s_{m} J x_{m}, x_{n}-x_{m}\right\rangle \leq 0 .
$$

Then $\left\{\left\|x_{n}\right\|\right\}$ is a nondecreasing sequence. If it is bounded, then $x_{n} \rightarrow$ (some) $x \in$ $X$.

This lemma follows easily from Lemma 1.4 of [4].

For facts about the theory of monotone operators the reader is referred to Barbu [3], Browder [5], Pascali and Sburlan [35] and Zeidler [40].

\section{ZEROS OF MAXIMAL MONOTONE OPERATORS}

We start with the following fundamental lemma.

Lemma 1. Let $T: X \supset D(T) \rightarrow 2^{X^{*}}$ be maximal monotone. Then, for every $x_{0}^{*} \in X^{*}$, the mapping $g: R_{+} \ni t \rightarrow(t T+J)^{-1} x_{0}^{*} \in X$ is continuous on $(0, \infty)$. If $x_{0}^{*}=J x_{0}$, for some $x_{0} \in D(T)$, then $g$ is also continuous at $t=0$. Actually, in this case we have $g(t) \rightarrow x_{0}$ as $t \rightarrow 0^{+}$.

Proof. The proof for the interval $(0, \infty)$ is contained in the proof of Theorem 12 by Guan and the author [12] for the case in which $X$ is uniformly convex and $0 \in D(T)$. This is why we present the proof for the present setting. We show first that the mapping $g$ is continuous at any $t_{0}>0$. To this end, we let

$$
x_{t}=(t T+J)^{-1} x_{0}^{*}, x_{t_{0}}=\left(t_{0} T+J\right)^{-1} x_{0}^{*}, \quad t \in(a, b),
$$


where $(a, b)$ is a finite subinterval of $(0, \infty)$ containing $t_{0}$. We shall prove that $x_{t} \rightarrow x_{t_{0}}$ as $t \rightarrow t_{0}$. We have

$$
x_{0}^{*}=t y_{t}^{*}+J x_{t}=t_{0} y_{t_{0}}^{*}+J x_{t_{0}}, \text { where } y_{t}^{*} \in T x_{t}, y_{t_{0}}^{*} \in T x_{t_{0}} .
$$

This implies

$$
\begin{aligned}
0 & =\left\langle\left(t y_{t}^{*}+J x_{t}\right)-\left(t_{0} y_{t_{0}}^{*}+J x_{t_{0}}\right), x_{t}-x_{t_{0}}\right\rangle \\
& =t\left\langle y_{t}^{*}-y_{t_{0}}^{*}, x_{t}-x_{t_{0}}\right\rangle+\left(t-t_{0}\right)\left\langle y_{t_{0}}^{*}, x_{t}-x_{t_{0}}\right\rangle+\left\langle J x_{t}-J x_{t_{0}}, x_{t}-x_{t_{0}}\right\rangle .
\end{aligned}
$$

To show that $\left\{x_{t}\right\}$ is bounded, we observe that (1) implies

$$
\left(\left\|x_{t}\right\|-\left\|x_{t_{0}}\right\|\right)^{2} \leq\left|t-t_{0}\right|\left\|y_{t_{0}}^{*}\right\|\left(\left\|x_{0}\right\|+\left\|x_{0}^{*}\right\|\right) .
$$

This inequality shows the boundedness of $\left\{x_{t}\right\}$, with its bound depending on a bound for $\left|t-t_{0}\right|(t \in(a, b))$ and $x_{t_{0}}$. Here we have used the fact that

$$
\langle J x-J y, x-y\rangle \geq(\|x\|-\|y\|)^{2} .
$$

Letting $K$ be such an upper bound for $\left\{x_{t}\right\}$, we obtain from (1) that

$$
\left\langle J x_{t}-J x_{t_{0}}, x_{t}-x_{t_{0}}\right\rangle \leq\left(K+\left\|x_{t_{0}}\right\|\right)\left|t-t_{0}\right|\left\|y_{t_{0}}^{*}\right\| .
$$

By Lemma B, $x_{t} \rightarrow x_{t_{0}}$.

Note that the above proof does not go through for $t_{0}=0$, unless $x_{0}^{*}=J x_{0}$, where $x_{0} \in D(T)$. This is because the point $x_{t_{0}}$ might not belong to $D(T)$ for the given functional $x_{0}^{*}$. This remark covers the case $t_{0}=0$. We observe that in this case $g(0)=x_{0}$.

The following proposition contains two necessary and sufficient conditions for the existence of a zero of a maximal monotone operator $T$.

Proposition 1. Let $T: X \supset D(T) \rightarrow 2^{X^{*}}$ be maximal monotone. Then the following statements are equivalent.

(a) $0 \in R(T)$;

(b) there exist an open and bounded set $G \subset X$ and a point $x_{0} \in G \cap D(T)$ such that

$$
\left\langle x^{*}, x-x_{0}\right\rangle \geq 0, \quad\left(x, x^{*}\right) \in(\partial G \cap D(T)) \times T x ;
$$

(c) there exist an open and bounded set $G \subset X$ and a point $x_{0} \in G \cap D(T)$ such that

$$
T x \not \supset \mu\left(J x-J x_{0}\right),(\mu, x) \in(-\infty, 0) \times(\partial G \cap D(T)) .
$$

Proof. We are going to show that (a) $\Rightarrow$ (b) $\Rightarrow$ (c) $\Rightarrow$ (a). We assume (a). Let $0 \in T x_{0}$, for some $x_{0} \in D(T)$. Then, given an open set $G$ containing $x_{0}$, we have, by monotonicity,

$$
\left\langle x^{*}, x-x_{0}\right\rangle \geq 0,\left(x, x^{*}\right) \in(\partial G \cap D(T)) \times T x,
$$

which is the boundary condition in (b). Thus (a) $\Rightarrow(\mathrm{b})$.

We assume (b) and show that (c) holds with the same set $G$ and point $x_{0} \in$ $G \cap D(T)$. Let (c) be false. Then there exist a number $\mu \in(-\infty, 0)$ and a point $x \in \partial G \cap D(T)$ such that

$$
x^{*}=\mu\left(J x-J x_{0}\right), \text { for some } x^{*} \in T x .
$$

Thus,

$$
0 \leq\left\langle x^{*}, x-x_{0}\right\rangle=\mu\left\langle J x-J x_{0}, x-x_{0}\right\rangle<0,
$$


where we have used (b) and the fact that $J$ is strictly monotone. Thus, (b) $\Rightarrow$ (c).

To show that (c) $\Rightarrow(\mathrm{a})$, we assume (c) and let $t=-\mu$. We observe that the mapping

$$
g: R_{+} \ni t \rightarrow(t T+J)^{-1} J x_{0} \in D(T)
$$

is continuous by Lemma 1 . We also observe that (c) says that

$$
g(t) \notin \partial G \text {, for any } t \in(0, \infty),
$$

i.e., $g(t)$ does not cross the boundary of the set $G$ for any positive value of $t$. Since $g(0)=x_{0} \in G$ and $g(t)$ is continuous, we conclude that $g(t)$ lies in the bounded, open set $G$ for all $t \in R_{+}$. Let $K>0$ be such that $\|g(t)\| \leq K, t \in R_{+}$, and let $\left\{t_{n}\right\} \subset(0, \infty)$ be such that $t_{n} \rightarrow \infty$ as $n \rightarrow \infty$. Then if $x_{n}=\left(t_{n} T+J\right)^{-1} J x_{0}$, we have $\left\|x_{n}\right\| \leq K$ and

$$
t_{n} T x_{n}+J x_{n} \ni J x_{0}, \text { for all } n=1,2, \ldots .
$$

Thus, for some sequence of functionals $x_{n}^{*} \in T x_{n}$, we have

$$
\left\|x_{n}^{*}\right\|=\left(1 / t_{n}\right)\left\|J x_{n}-J x_{0}\right\| \leq\left(1 / t_{n}\right)\left(K+\left\|x_{0}\right\|\right) \rightarrow 0 \text { as } n \rightarrow \infty .
$$

Since $X$ is reflexive, we may assume that $x_{n} \rightarrow \widetilde{x}_{0} \in X$ as $n \rightarrow \infty$. Since

$$
\lim _{n \rightarrow \infty}\left\langle x_{n}^{*}, x_{n}-\widetilde{x}_{0}\right\rangle=0,
$$

we can apply Lemma A to conclude that $\widetilde{x}_{0} \in D(T)$ and $0 \in T \widetilde{x}_{0}$.

This result has a good number of corollaries. The following one is an improvement of Theorem 1 in Yang's paper [39]. Yang used Browder's degree in [6]. For a set $A \subset X^{*}$ we set $|A|=\inf \left\{\left\|x^{*}\right\|: x^{*} \in A\right\}$.

Corollary 1. Let $T: X \supset D(T) \rightarrow 2^{X^{*}}$ be maximal monotone. Assume that there exist an open, bounded set $G \subset X$ and a set $A \subset X^{*}$ such that

$$
|A|<r \leq|T x|, x \in \partial G \cap D(T),
$$

where $r$ is a constant. Assume, further, that there exist $x_{0} \in G \cap D(T)$ and $k \in$ $[0, r-|A|)$ such that

$$
\left\langle x^{*}-a^{*}, x-x_{0}\right\rangle \geq-k\left\|x-x_{0}\right\| \text {, for every } x \in \partial G \cap D(T), x^{*} \in T x, a^{*} \in A .
$$

Then $B_{r}(0) \subset R(T)$.

Proof. We may assume that $x_{0}=0$; otherwise we consider instead of $T$ the operator $\widetilde{T}(x) \equiv T\left(x+x_{0}\right), x \in D(\widetilde{T})=D(T)-x_{0}$. We show first that $B_{q}(0)$ lies in the range of $T$, where $q=[r-(|A|+k)] / 2$. This was done in the proof of Theorem 1 of the author in [17], where $T$ was assumed to be a single-valued $m$-accretive operator, $A=T(0)$ and $k=0$. To this end, we fix $p^{*} \in B_{q}(0)$ and observe that it suffices to show that condition (c) of Proposition 1 is satisfied for the operator $\widetilde{T} x \equiv T x-p^{*}$ and $x_{0}=0$. Let us assume that $\mu J \widetilde{x} \in \widetilde{T} \widetilde{x}$ for some $\mu<0$ and some $\widetilde{x} \in \partial G \cap D(T)$. Then,

$$
\widetilde{x}^{*}+t J \widetilde{x}=p^{*}, \text { for some } x^{*} \in T \widetilde{x},
$$

where $t=-\mu$. We fix $a_{0}^{*} \in A$ and use the monotonicity of $T$ to obtain

$$
\left\langle x^{*}-a_{0}^{*}, \widetilde{x}\right\rangle+\left\langle a_{0}^{*}, \widetilde{x}\right\rangle+t\langle J \widetilde{x}, \widetilde{x}\rangle=\left\langle p^{*}, \widetilde{x}\right\rangle .
$$

This easily implies that

$$
t\|J \widetilde{x}\| \leq\left\|a_{0}^{*}\right\|+\left\|p^{*}\right\|+k .
$$


Since $a_{0}^{*}$ is arbitrary in $A$, we actually have

$$
t\|J \widetilde{x}\| \leq|A|+\left\|p^{*}\right\|+k .
$$

On the other hand, since $|T \widetilde{x}| \geq r$, we have

$$
r-\left\|p^{*}\right\| \leq|T \widetilde{x}|-\left\|p^{*}\right\| \leq\left\|\widetilde{x}^{*}\right\|-\left\|p^{*}\right\| \leq\left\|\widetilde{x}^{*}-p^{*}\right\|=t\|J \widetilde{x}\| \leq|A|+\left\|p^{*}\right\|+k .
$$

This says that $\left\|p^{*}\right\| \geq[r-(|A|+k)] / 2=q$, which is a contradiction. Thus, by Proposition $1, B_{q}(0) \subset R(T)$.

The rest of the proof actually follows as in Parts 2 and 3 of Theorem 1 in [17]. However, since Kirk and Schöneberg [29, Theorem 3] have shortened that proof considerably, we give the rest of the proof following their method. We let $p^{*} \in B_{r}(0)$ and

$$
M=\left\{t \in[0,1]: t p^{*} \in R(T)\right\} .
$$

We know that $M \neq \emptyset$ by the above part of the proof. We let $t_{0}=\sup M$ and show that $t_{0}=1$. Let $t_{0}<1$ and let $\left\{t_{n}\right\} \subset M$ with $t_{n} \rightarrow t_{0}$. We let $x_{n} \in D(T)$ be such that $t_{n} p^{*} \in T x_{n}$. Let

$$
T_{n} x \equiv T\left(x+x_{n}\right)-t_{n} p^{*}, \quad x \in D\left(T_{n}\right) \equiv D(T)-x_{n} .
$$

Then $0 \in T_{n}(0)$ and, moreover,

$$
\left|T_{n} x\right| \geq r-t_{n}\left\|p^{*}\right\|, \quad x \in \partial G_{n} \cap D\left(T_{n}\right),
$$

where $G_{n} \equiv G-x_{n}$. Applying again the first part of this proof, with $A=$ $T_{n}(0),|A|=0, k=0$ and $r-t_{n}\left\|p^{*}\right\|$ in place of $r$, we get

$$
B_{\widetilde{q}}(0) \subset R(T),
$$

where $\widetilde{q}=\left(r-t_{n}\left\|p^{*}\right\|\right) / 2$. Choosing $t \in\left(t_{0}, 1\right)$ sufficiently close to $t_{0}$, we may also select a positive integer $n$ such that

$$
\left(t-t_{n}\right)\left\|p^{*}\right\|<\left(r-t_{n}\left\|p^{*}\right\|\right) / 2 .
$$

This implies that there exists some $y_{n} \in D\left(T_{n}\right)$ such that $\left(t-t_{n}\right) p^{*} \in T_{n} y_{n}=$ $T\left(y_{n}+x_{n}\right)-t_{n} p^{*}$. Thus, $t p^{*} \in T\left(y_{n}+x_{n}\right)$, which contradicts the maximum property of $t_{0}$. It follows that $t_{0}=1$. Since $t p^{*} \in R(T)$ for any $\left(t, p^{*}\right) \in[0,1) \times B_{r}(0)$, we have that $B_{r}(0) \subset R(T)$.

Corollary 2 below follows from Corollary 1, and it is Theorem 2 in Yang's paper [39].

Corollary 2. Let $T: X \supset D(T) \rightarrow 2^{X^{*}}$ be maximal monotone. Assume that there exists $x_{0} \in D(T)$ such that

$$
\left|T x_{0}\right|<r \leq \liminf _{\substack{\|x\| \rightarrow \infty \\ x \in D(T)}}|T x|
$$

Then $B_{r}(0) \subset R(T)$.

Proof. It is easy to see that there exist $\epsilon \in(0, r), \delta>0$ such that

$$
\left|T x_{0}\right|<r-\epsilon \leq|T x|, x \in \partial B_{\delta}\left(x_{0}\right) \cap D(T) .
$$

In order to apply Corollary 1 , we let $\widetilde{T} x=T\left(x+x_{0}\right), x \in D(\widetilde{T}) \equiv D(T)-x_{0}$, and $\widetilde{G}=B_{\delta}\left(x_{0}\right)-x_{0}=B_{\delta}(0)$. We observe that $0 \in \widetilde{G} \cap D(\widetilde{T})$, and let $A=\widetilde{T}(0)$. We also observe that

$$
|\widetilde{T}(0)|<r-\epsilon \leq|\widetilde{T} x|, \quad x \in \partial \widetilde{G} \cap D(\widetilde{T}) .
$$


Furthermore, given $a^{*} \in \widetilde{T}(0)$ and $x \in \partial \widetilde{G} \cap D(\widetilde{T})$, we have

$$
\left\langle x^{*}-a^{*}, x\right\rangle \geq 0, x^{*} \in \widetilde{T} x .
$$

The proof is complete because $B_{r-\epsilon}(0) \subset R(T)$ for every $\epsilon>0$.

Surprisingly enough, a version of Altman's well-known condition in [1], [2], namely,

$$
\left\|x^{*}\right\|^{2} \geq\left\|x^{*}-\left(J x-J x_{0}\right)\right\|^{2}-\left\|J x-J x_{0}\right\|^{2}, x \in \partial G \cap D(T), x^{*} \in T x,
$$

for some $x_{0} \in G$, implies the boundary condition (2).

Corollary 3. Let $T: X \supset D(T) \rightarrow 2^{X^{*}}$ be maximal monotone. Assume that there exist an open and bounded set $G \subset X$ and a point $x_{0} \in G \cap D(T)$ such that (3) is satisfied. Then $0 \in R(T)$.

Proof. We are going to show that (2) is satisfied. In fact, assume that

$$
T \widetilde{x} \ni \mu_{0}\left(J \widetilde{x}-J x_{0}\right),
$$

for some $\left(\mu_{0}, \widetilde{x}\right) \in(-\infty, 0) \times(\partial G \cap D(T))$. Then, for $x^{*}=\mu_{0}\left(J \widetilde{x}-J x_{0}\right) \in T \widetilde{x}$, we have

$$
\left\|x^{*}\right\|^{2} \geq\left\|x^{*}-\left(J \widetilde{x}-J x_{0}\right)\right\|^{2}-\left\|J \widetilde{x}-J x_{0}\right\|^{2}
$$

or

$$
\left\|\mu_{0}\left(J \widetilde{x}-J x_{0}\right)\right\|^{2} \geq\left(\mu_{0}-1\right)^{2}\left\|J \widetilde{x}-J x_{0}\right\|^{2}-\left\|J \widetilde{x}-J x_{0}\right\|^{2} .
$$

Since $\left\|J \widetilde{x}-J x_{0}\right\| \neq 0$, by the fact that $J$ is one-to-one, we have the contradiction $\mu_{0}^{2} \geq\left(\mu_{0}-1\right)^{2}-1$.

In particular, if we take $x_{0}=0$ in Corollary 3 , then the inequality in Altman's condition (3) is satisfied under either one of the following conditions:

(i) $\left\|x^{*}-J x\right\| \leq\left\|x^{*}\right\|, x \in \partial G \cap D(T), x^{*} \in T x$;

(ii) $\left\|x^{*}-J x\right\| \leq\|x\|, x \in \partial G \cap D(T), x^{*} \in T x$;

The next result is an extension of Theorem 5 of Reich and Torrejón to maximal monotone operators $T$.

Corollary 4. Let $T: X \supset D(T) \rightarrow 2^{X^{*}}$ be maximal monotone. Assume that there exist a positive constant $c$, an open, bounded set $G \subset X$ and a point $x_{0} \in G \cap D(T)$ such that

$$
\left\langle x^{*}, x-x_{0}\right\rangle \geq c, \text { for all }\left(x, x^{*}\right) \in(\partial G \cap D(T)) \times T x .
$$

Then $B_{c / r}(0) \subset R(T)$, where $r=\sup \left\{\left\|x-x_{0}\right\|: x \in \partial G\right\}$.

Proof. We let $\widetilde{T} x \equiv T x-p$, for a fixed point $p \in B_{c / r}(0)$. Using (4), we observe that, for any $x \in \partial G \bigcap D(T)$ and any $x^{*} \in T x$,

$$
\begin{aligned}
\left\langle x^{*}-p, x-x_{0}\right\rangle & =\left\langle x^{*}, x-x_{0}\right\rangle-\left\langle p, x-x_{0}\right\rangle \\
& \geq c-\|p\|\left\|x-x_{0}\right\| \geq c-\|p\| r>0 .
\end{aligned}
$$

Applying Proposition 1, we obtain a zero for the operator $\widetilde{T}$. This completes the proof.

The following theorem shows that a maximal monotone operator $T$ has a zero if and only if every orbit $(t T+J)^{-1} x^{*}, t \in(0, \infty)$, is bounded, and not just the orbits of the type $(t T+J)^{-1} J x_{0}$, where $x_{0} \in D(T)$. 
Theorem 1. Let $T: X \supset D(T) \rightarrow 2^{X^{*}}$ be maximal monotone. Then $0 \in R(T)$ if and only if

$$
\limsup _{t \rightarrow \infty}\left\|(t T+J)^{-1} x^{*}\right\|<+\infty, \text { for every } x^{*} \in X^{*} .
$$

Proof. Let (5) be satisfied. We choose $x^{*}=J x_{0}$, for some $x_{0} \in D(T)$, and pick an open set $G \subset X$ sufficiently large so that the orbit $g(t) \equiv(t T+J)^{-1} J x_{0}$, with $x_{0} \in G$, remains in $G$ for all $t>0$. Then

$$
\limsup _{t \rightarrow \infty}\left\|(t T+J)^{-1} J x_{0}\right\|<+\infty,
$$

and part (c) of Proposition 1 implies that $0 \in R(T)$.

Conversely, assume that $0 \in R(T)$. Then (6) holds by part (c) of Proposition 1. Fix $x^{*} \in X^{*}$ and let $\left\{t_{n}\right\} \subset(0, \infty)$ be such that $t_{n} \rightarrow \infty$ and $\left\|x_{1, n}\right\| \rightarrow \infty$, where

$$
x_{1, n}=\left(t_{n} T+J\right)^{-1} x^{*} \text {. }
$$

Let

$$
x_{2, n}=\left(t_{n} T+J\right)^{-1} x_{0}^{*},
$$

where $x_{0}^{*}=J x_{0}$. Then, for some $y_{1, n}^{*} \in T\left(x_{1, n}\right), y_{2, n}^{*} \in T\left(x_{2, n}\right)$,

$$
x^{*}=t_{n} y_{1, n}^{*}+J x_{1, n} \text { and } x_{0}^{*}=t_{n} y_{2, n}^{*}+J x_{2, n} .
$$

We have

$$
\begin{aligned}
\left\langle x^{*}-x_{0}^{*}, x_{1, n}-x_{2, n}\right\rangle & =t_{n}\left\langle y_{1, n}^{*}-y_{2, n}^{*}, x_{1, n}-x_{2, n}\right\rangle+\left\langle J x_{1, n}-J x_{2, n}, x_{1, n}-x_{2, n}\right\rangle \\
& \geq\left(\left\|x_{1, n}\right\|-\left\|x_{2, n}\right\|\right)^{2},
\end{aligned}
$$

which implies

$$
\left(\left\|x_{1, n}\right\|-\left\|x_{2, n}\right\|\right)^{2} \leq\left\|x^{*}-x_{0}^{*}\right\|\left\|x_{1, n}-x_{2, n}\right\| .
$$

Since $\left\{x_{2, n}\right\}$ is bounded, so is $\left\{x_{1, n}\right\}$ by the above inequality, i.e., a contradiction. It follows that (5) is true, and the proof is complete.

A natural question to ask here is whether a statement like Theorem 1 is actually true for the Yosida resolvents $J_{t}, t \in(0, \infty)$, defined in the introduction. The following result gives an affirmative answer to this question.

Theorem 2. Let $T: X \supset D(T) \rightarrow 2^{X^{*}}$ be maximal monotone. Then $0 \in R(T)$ if and only if

$$
\limsup _{t \rightarrow \infty}\left\|J_{t} x\right\|<+\infty, \text { for every } x \in X .
$$

Proof. We assume that (7) is satisfied for some (fixed) $x \in X$, and show that $0 \in R(T)$. Let $\left\{t_{n}\right\} \subset(0, \infty)$ be such that $t_{n} \rightarrow \infty$ as $n \rightarrow \infty$. We have

$$
T_{t_{n}} x=\left(1 / t_{n}\right) J\left(x-J_{t_{n}} x\right)
$$

and

$$
\left\|T_{t_{n}} x\right\|=\left(1 / t_{n}\right)\left\|x-J_{t_{n}} x\right\| .
$$

Since $\left\{J_{t_{n}} x\right\}$ is bounded, $\left\|T_{t_{n}} x\right\| \rightarrow 0$ as $n \rightarrow \infty$. However, $T_{t_{n}} x \in T J_{t_{n}} x$. If we let, without loss of generality, $J_{t_{n}} x \rightarrow \widetilde{x} \in X$, then Lemma A implies that $\widetilde{x} \in D(T)$ and $0 \in T \widetilde{x}$. 
To show that (7) is necessary, let $y \in T^{-1}(0)$. Then we have

$$
\begin{aligned}
0 & \leq\left\langle T_{t} x, J_{t} x-y\right\rangle=-(1 / t)\left\langle J\left(J_{t} x-x\right), J_{t} x-y\right\rangle \\
& =-(1 / t)\left\langle J\left(J_{t} x-x\right), J_{t} x-x\right\rangle-(1 / t)\left\langle J\left(J_{t} x-x\right), x-y\right\rangle,
\end{aligned}
$$

where we have used the fact that $T_{t} y=0$ and that $T_{t}$ is a monotone operator. From (8) we obtain

$$
\left\|J_{t} x-x\right\|^{2} \leq-\left\langle J\left(J_{t} x-x\right), x-y\right\rangle \leq\left\|J_{t} x-x\right\|\|x-y\|,
$$

and, eventually, the boundedness of $\left\{\left\|J_{t} x-x\right\|\right\}, t \in(0, \infty)$. This yields the boundedness of $\left\{\left\|J_{t} x\right\|\right\}, t \in(0, \infty)$.

An easy consequence of Theorems 1 and 2 is the following corollary about the surjectivity of a maximal monotone operator $T$.

Corollary 5. A maximal monotone operator $T: X \supset D(T) \rightarrow 2^{X^{*}}$ is surjective if and only if one of the following statements holds.

(i) for every $p^{*}, x^{*} \in X^{*}$ we have

$$
\limsup _{t \rightarrow \infty}\left\|\left(t\left(T-p^{*}\right)+J\right)^{-1} x^{*}\right\|<+\infty ;
$$

(ii) for every $p^{*} \in X^{*}, x \in X$ we have

$$
\limsup _{t \rightarrow \infty}\left\|J_{t}^{p^{*}} x\right\|<+\infty
$$

where $J_{t}^{p^{*}}$ is the Yosida resolvent of the operator $x \rightarrow T x-p^{*}$.

It is well-known that a maximal monotone operator $T$ is surjective if and only if $T^{-1}$ is locally bounded (cf. Pascali and Sburlan [35, p. 147]). However, Corollary 5 does not make direct use of the concept of local boundedness. Case (i) there, for example, says that $T$ is surjective if and only if for every $p^{*}, x^{*} \in X^{*}$ the set $\left\{x_{t}: t \in(0, \infty)\right\}$ of solutions of the problem

$$
t\left(T x-p^{*}\right)+J x \ni x^{*}
$$

is bounded. Inclusion (9) has exactly one solution $x_{t}$ for each $t>0$.

We now take Theorems 1 and 2 one step further. We show that the limits of the orbits in Theorem 1 and the resolvents in Theorem 2 exist as $t \rightarrow \infty$ and lie in the set $T^{-1}(0)$.

Theorem 3. Let $T: X \supset D(T) \rightarrow 2^{X^{*}}$ be maximal monotone. Then $0 \in R(T)$ if and only if for every $x^{*} \in X^{*}$ and $x \in X$ the limits

$$
\lim _{t \rightarrow \infty}(t T+J)^{-1} x^{*} \text { and } \lim _{t \rightarrow \infty} J_{t} x
$$

exist and belong to the set $T^{-1}(0)$. Furthermore, $T$ is surjective if and only if for every $p^{*}, x^{*} \in X^{*}$ and $x \in X$ the limits

$$
\lim _{t \rightarrow \infty}\left(t\left(T-p^{*}\right)+J\right)^{-1} x^{*} \text { and } \lim _{t \rightarrow \infty} J_{t}^{p^{*}} x
$$

exist, where $J_{t}^{p^{*}}$ is the Yosida resolvent of the operator $x \rightarrow T x-p^{*}$.

Proof. We only need to show the necessity of the two limits in (10). Let $\left\{t_{n}\right\} \subset$ $(0, \infty)$ be such that $t_{n} \rightarrow \infty$ as $n \rightarrow \infty$. Let

$$
x_{n}=\left(t_{n} T+J\right)^{-1} x^{*}, \text { for some } x^{*} \in X^{*} .
$$


From the proof of Proposition 1 we know that $\left\{x_{n}\right\}$ has a subsequence converging weakly to a zero of $T$. We denote this subsequence again by $\left\{x_{n}\right\}$ and let $x_{n} \rightarrow$ $x_{0} \in T^{-1}(0)$. We show first that $x_{n} \rightarrow x_{0}$. To this end, we observe that, for some $x_{n}^{*} \in T x_{n}$, we have

$$
t_{n}\left\langle x_{n}^{*}, x_{n}-x_{0}\right\rangle+\left\langle J x_{n}-J x_{0}, x_{n}-x_{0}\right\rangle=\left\langle x^{*}-J x_{0}, x_{n}-x_{0}\right\rangle,
$$

which gives

$$
0 \leq \limsup _{n \rightarrow \infty}\left\langle J x_{n}-J x_{0}, x_{n}-x_{0}\right\rangle \leq \lim _{n \rightarrow \infty}\left\langle x^{*}-J x_{0}, x_{n}-x_{0}\right\rangle=0 .
$$

By Lemma B, we have $x_{n} \rightarrow x_{0}$ as $n \rightarrow \infty$. We now show that if $\left\{x_{k}\right\}$ is another sequence, corresponding to some sequence $\left\{t_{k}\right\} \subset(0, \infty)$ with $t_{k} \rightarrow \infty$ as $k \rightarrow \infty$, and satisfying $x_{k} \rightarrow x_{1}$, we have $x_{1}=x_{0}$. In fact, from (11) we get

$$
\left\langle J x_{n}-x^{*}, x_{n}-x_{1}\right\rangle \leq 0,
$$

because (11) holds for any $x_{0} \in T^{-1}(0)$. This implies

$$
\left\langle J x_{0}-x^{*}, x_{0}-x_{1}\right\rangle \leq 0 .
$$

Similarly, by considering (11) for the sequences $\left\{t_{k}\right\}$ and $\left\{x_{k}\right\}$, and then taking the limits as $k \rightarrow \infty$, we get

$$
\left\langle J x_{1}-x^{*}, x_{1}-x_{0}\right\rangle \leq 0
$$

or

$$
\left\langle x^{*}-J x_{1}, x_{0}-x_{1}\right\rangle \leq 0
$$

Adding (12) and (13), we get

$$
\left\langle J x_{0}-J x_{1}, x_{0}-x_{1}\right\rangle \leq 0,
$$

which, by the strict monotonicity of $J$, implies that $x_{1}=x_{0}$. We have shown the following statement: every sequence $\left\{t_{n}\right\} \subset(0, \infty)$ with $t_{n} \rightarrow \infty$ contains a subsequence $\left\{t_{n_{k}}\right\}$ such that

$$
\lim _{k \rightarrow \infty}\left(t_{n_{k}} T+J\right)^{-1} x^{*}=x_{0},
$$

where $x_{0} \in T^{-1}(0)$ is independent of the sequence $\left\{t_{n_{k}}\right\}$. This says that the first limit in (10) exists and lies in $T^{-1}(0)$.

To show the necessity of the second limit in (10), we fix $x \in X$ and let $x_{n}=J_{t_{n}} x$, where $\left\{t_{n}\right\}$ is as above. We also assume that $x_{n} \rightarrow x_{0} \in T^{-1}(0)$, by the proof of Theorem 2. We show first that $x_{n} \rightarrow x_{0}$. To this end, we observe that

$$
t_{n} T_{t_{n}} x=J\left(x-J_{t_{n}} x\right)=J\left(x-x_{n}\right) \in t_{n} T J_{t_{n}} x=t_{n} T x_{n} .
$$

Thus, for some $y_{n}^{*} \in T x_{n}$,

$$
t_{n} y_{n}^{*}=J\left(x-x_{n}\right)
$$

We have

$$
\begin{aligned}
0 & \leq t_{n}\left\langle y_{n}^{*}, x_{n}-x_{0}\right\rangle=\left\langle J\left(x-x_{n}\right), x_{n}-x_{0}\right\rangle \\
& =\left\langle J\left(x-x_{n}\right)-J\left(x-x_{0}\right), x_{n}-x_{0}\right\rangle+\left\langle J\left(x-x_{0}\right), x_{n}-x_{0}\right\rangle \\
& =-\left\langle J\left(x_{n}-x\right)-J\left(x_{0}-x\right), x_{n}-x_{0}\right\rangle+\left\langle J\left(x-x_{0}\right), x_{n}-x_{0}\right\rangle \\
& \leq-\left(\left\|x_{n}-x\right\|-\left\|x_{0}-x\right\|\right)^{2}+\left\langle J\left(x-x_{0}\right), x_{n}-x_{0}\right\rangle
\end{aligned}
$$


which implies

$$
\left(\left\|x_{n}-x\right\|-\left\|x_{0}-x\right\|\right)^{2} \leq\left\langle J\left(x-x_{0}\right), x_{n}-x_{0}\right\rangle .
$$

Thus, $\left\|x_{n}-x\right\| \rightarrow\left\|x_{0}-x\right\|$. Since $X$ is locally uniformly convex and $x_{n} \rightarrow x_{0}$, we have that $x_{n} \rightarrow x_{0}$.

Now, we show that $x_{0}$ is independent of the sequence $\left\{t_{n}\right\}$. Assume that $\left\{x_{k}\right\}$ is a sequence corresponding to $\left\{t_{k}\right\}$ with $\left\{t_{k}\right\} \subset(0, \infty), t_{k} \rightarrow \infty$ and $x_{k} \rightarrow x_{1} \in T^{-1}(0)$. From (14) we get

$$
0 \leq\left\langle J\left(x-x_{n}\right), x_{n}-x_{1}\right\rangle,
$$

because the first line of (14) holds for $x_{1} \in T^{-1}(0)$ instead of $x_{0}$. Similarly, we have

$$
0 \leq\left\langle J\left(x-x_{k}\right), x_{k}-x_{0}\right\rangle \text {. }
$$

Thus,

$$
\left\langle J\left(x-x_{0}\right), x_{0}-x_{1}\right\rangle \geq 0
$$

or

$$
\left\langle-J\left(x-x_{0}\right), x_{1}-x_{0}\right\rangle \geq 0
$$

and

$$
\left\langle J\left(x-x_{1}\right), x_{1}-x_{0}\right\rangle \geq 0 .
$$

Adding (15) and (16) and using the strict monotonicity of $J$, we get $x_{1}=x_{0}$. Thus, our assertion follows as in the first part of the proof.

\section{ZEROS OF DEMICONTINUOUS MONOTONE OPERATORS}

We demonstrate now how our considerations of Section 2 can be applied to mappings $T$ of other monotone types. A mapping $T: X \supset D(T) \rightarrow X^{*}$ is said to be of type $(S)$ if for any sequence $\left\{x_{n}\right\} \subset D(T)$ such that $x_{n} \rightarrow x \in X$ and

$$
\lim _{n \rightarrow \infty}\left\langle T x_{n}, x_{n}-x\right\rangle=0
$$

we have $x_{n} \rightarrow x$. A mapping $T: X \supset \bar{G} \rightarrow X^{*}, G$ open, is said to be of type $\left(S_{q}\right)$ if for every sequence $\left\{x_{n}\right\} \subset G$ such that $x_{n} \rightarrow x \in X$ and $T x_{n} \rightarrow 0$ we have $0 \in R(T)$.

Obviously, a demicontinuous mapping $T: X \supset \bar{G} \rightarrow X^{*}, G$ open, of type $(S)$ is also of type $\left(S_{q}\right)$. We need the following result, essentially due to Park [34, Theorem 3]. A property $\mathcal{P}$ holds "locally" in an open set $G$ if every point in $G$ has a neighborhood in which the property $\mathcal{P}$ holds.

Theorem A. Let $G \subset X$ be open and let $T: G \rightarrow X^{*}$ be demicontinuous, locally monotone, locally injective and locally of type $(S)$. Then $T(G)$ is open.

Theorem 4. Let $T: X \supset \bar{G} \rightarrow X^{*}$ be demicontinuous, monotone and of type $\left(S_{q}\right)$, where $G$ is open and bounded. Let $0 \notin T(\partial G)$. Then the following statements are equivalent:

(a) $0 \in T(G)$;

(b) there exists $x_{0} \in G$ such that

$$
\left\langle T x, x-x_{0}\right\rangle \geq 0, x \in \partial G ;
$$


(c) there exists $x_{0} \in G$ such that

$$
T x \neq \mu\left(J x-J x_{0}\right),(\mu, x) \in(-\infty, 0) \times \partial G .
$$

Proof. The proof of (a) $\Rightarrow(\mathrm{b}) \Rightarrow$ (c) follows as in the proof of Proposition 1. We only show that (c) $\Rightarrow$ (a). We fix $\alpha>0$ and show first that

$$
g_{t}(x) \equiv t\left(T x+\alpha\left(J x-J x_{0}\right)\right)+J x-J x_{0}=0
$$

has a solution $x \in G$ for each $t \in R_{+}$. To this end, we note first that $g_{t}, t \in R_{+}$, is a demicontinuous, monotone and injective operator of type $(S)$ on every closed ball contained in $G$. We let

$$
K_{G}=\sup \{\|x\|: x \in G\} .
$$

The set

$$
S=\left\{t \in R_{+}: g_{t}(x)=0, \text { for some } x \in G\right\}
$$

equals $R_{+}$. In fact, $S$ is open and closed in the interval $R_{+}$. To show that it is closed, fix $t \in R_{+}$and let $\left\{t_{n}\right\} \subset S$ be such that $t_{n} \rightarrow t$. If $t=0$, then $g_{t}(x)=0$ implies $x=x_{0}$ and we are done. We assume that $t>0$. In this case $\left\{t_{n}\right\}$ is bounded away from zero, and

$$
t_{n}\left(T x_{n}+\alpha\left(J x_{n}-J x_{0}\right)\right)+J x_{n}-J x_{0}=0
$$

implies

$$
\begin{aligned}
t_{n}\left\|T x_{n}\right\| & =\left\|\left(1+\alpha t_{n}\right)\left(J x_{n}-J x_{0}\right)\right\| \leq\left(1+\alpha t_{n}\right)\left(\left\|x_{n}\right\|+\left\|x_{0}\right\|\right) \\
& \leq\left(1+\alpha t_{n}\right)\left(K_{G}+\left\|x_{0}\right\|\right),
\end{aligned}
$$

and the boundedness of $\left\{\left\|T x_{n}\right\|\right\}$. Let $L>0$ be such an upper bound. We have

$$
\begin{aligned}
0= & t_{n}\left\langle T x_{n}-T x_{m}, x_{n}-x_{m}\right\rangle+\left(t_{n}-t_{m}\right)\left\langle T x_{m}, x_{n}-x_{m}\right\rangle \\
& +\alpha t_{n}\left\langle J x_{n}-J x_{m}, x_{n}-x_{m}\right\rangle+\alpha\left(t_{n}-t_{m}\right)\left\langle J x_{m}-J x_{0}, x_{n}-x_{m}\right\rangle \\
& +\left\langle J x_{n}-J x_{m}, x_{n}-x_{m}\right\rangle \\
\geq & \left(t_{n}-t_{m}\right)\left\langle T x_{m}, x_{n}-x_{m}\right\rangle+\alpha\left(t_{n}-t_{m}\right)\left\langle J x_{m}-J x_{0}, x_{n}-x_{m}\right\rangle \\
& +\left\langle J x_{n}-J x_{m}, x_{n}-x_{m}\right\rangle,
\end{aligned}
$$

from which we obtain

$$
\begin{aligned}
\left\langle J x_{n}-J x_{m}, x_{n}-x_{m}\right\rangle & \leq\left|t_{n}-t_{m}\right|\left(\left\|T x_{m}\right\|+\alpha\left\|J x_{m}-J x_{0}\right\|\right)\left\|x_{n}-x_{m}\right\| \\
& \leq 2\left|t_{n}-t_{m}\right|\left[L+\alpha\left(K_{G}+\left\|x_{0}\right\|\right)\right] K_{G},
\end{aligned}
$$

and

$$
0 \leq \limsup _{m, n \rightarrow \infty}\left\langle J x_{n}-J x_{m}, x_{n}-x_{m}\right\rangle \leq 0 .
$$

Since $\left\{x_{n}\right\}$ and $\left\{J x_{n}\right\}$ are bounded sequences, we may assume that $x_{n} \rightarrow x$ and $J x_{n} \rightarrow x^{*}$ as $n \rightarrow \infty$. By Lemma A, $x_{n} \rightarrow x$. Using the demicontinuity of $T$ and the continuity of $J$, we obtain

$$
t\left(T x+\alpha\left(J x-J x_{0}\right)\right)+J x-J x_{0}=0 .
$$

Since

$$
T x=-(\alpha+(1 / t))\left(J x-J x_{0}\right),
$$

the boundary condition (17) precludes $x$ from belonging to $\partial G$. Thus, $x \in G$ and $t \in S$. This concludes the proof that $S$ is closed. 
To show that $S$ is open, we assume that there are $t \in S$ and $\left\{t_{n}\right\} \subset R_{+}$such that $t_{n} \rightarrow t$ and $g_{t_{n}}(x) \neq 0$ for every $x \in G$. It is easy to see that $t_{n}>0$. We also have $g_{t}(\widetilde{x})=0$, for some $x \in G$. Since $G$ is open, there exists a ball $B \equiv B_{r}(\widetilde{x})$ such that $\bar{B} \subset G$. Since $T$ is locally bounded, we may assume that $T \bar{B}$ is a bounded set. We let $y_{n}=g_{t_{n}}(\widetilde{x}) \in g_{t_{n}}(B)$. Since $0 \notin g_{t_{n}}(B)$, the line segment $\left[0, y_{n}\right] \equiv\left\{\lambda y_{n}: \lambda \in[0,1]\right\}$ cannot lie entirely inside the set $g_{t_{n}}(B)$, which is open by Theorem A. Thus, there exists $v_{n} \in\left[0, y_{n}\right] \cap \partial g_{t_{n}}(B)$. Since $g_{t}(\bar{B})$ is closed, it is easy to see that $\partial g_{t_{n}}(B) \subset g_{t_{n}}(\partial B)$. Thus, $v_{n}=g_{t_{n}}\left(x_{n}\right)$ with $x_{n} \in \partial B$. Since $v_{n} \rightarrow 0$ as $n \rightarrow \infty\left(g_{t_{n}}(\widetilde{x}) \rightarrow g_{t}(\widetilde{x})=0\right)$, we obtain from

$$
t_{n}\left(T x_{n}+\alpha\left(J x_{n}-J x_{0}\right)\right)+J x_{n}-J x_{0}=v_{n}
$$

and a relation like (20), that (21) holds and, eventually, $x_{n} \rightarrow x \in \partial G$. Since

$$
t\left(T x+\alpha\left(J x-J x_{0}\right)\right)+J x-J x_{0}=0,
$$

we have a contradiction to the boundary condition (17) for $t \neq 0$. If $t=0$, then $J x=J x_{0}$ implies that $x=x_{0}$, i.e., a contradiction again. It follows that $S$ is open in $R_{+}$. We have shown that $S=R_{+}$. This says that given an increasing sequence $\left\{t_{n}\right\} \subset(0, \infty)$ with $t_{n} \rightarrow \infty$, there exists a sequence of vectors $x_{n} \in G$ such that (19) holds. We easily obtain

$$
v_{n} \equiv T x_{n}+\alpha\left(J x_{n}-J x_{0}\right) \rightarrow 0 \text { as } n \rightarrow \infty .
$$

This relation leads again to (21) and the fact that $x_{n} \rightarrow$ (some) $\widetilde{x} \in \bar{G}$. We must have $\widetilde{x} \in G$; otherwise the boundary condition (17) is violated. It follows that we have a solution $x=x_{\alpha} \in G$ of the equation

$$
T x+\alpha\left(J x-J x_{0}\right)=0
$$

for every $\alpha>0$. Let $\alpha=(1 / n)$ and $x_{n}=x_{1 / n}$. Since $\left\{x_{n}\right\}$ lies in the bounded set $G$, we have that $T x_{n} \rightarrow 0$ as $n \rightarrow \infty$. Assuming that $x_{n} \rightarrow \widetilde{x} \in X$ and taking into consideration that $T$ is of type $\left(S_{q}\right)$ and $0 \notin T(\partial G)$, we have that $0 \in T(G)$. The proof is complete.

Theorem 4 may be compared to Theorem 7 of Guan [10], where another boundary condition is given for a set $G$ which is also assumed to be convex, and $T$ is of type $(S)$. Such a mapping satisfies condition $\left(S_{q}\right)$ automatically. This is due to the fact that in this case $x_{n} \rightarrow x$ and $T x_{n} \rightarrow 0$ imply that $x \in \bar{G}$ and $T x_{n} \rightarrow T x=$ 0 . Theorem 4 is also an extension of Theorem 3 of Morales [31], where $T$ is a demicontinuous, strongly accretive operator.

The next result improves Theorem 1 of Guan [10] and Theorem 2 of Park [34]. Guan assumed that the set $G$ is also convex and Park assumed that $X$ is a real Hilbert space. Results of this type are quite basic for the study of the ranges of nonlinear operators. The reason for this is that a proper choice of the pathwise connected set $M$ produces criteria for the existence of zeros of such operators. A set $M \subset X^{*}$ is called "pathwise connected" if for every $x^{*}, y^{*} \in M$ there exists a continuous function $s:[0,1] \rightarrow M$ such that $s(0)=x^{*}$ and $s(1)=y^{*}$.

Proposition 2. Let $T: X \supset \bar{G} \rightarrow X^{*}$ be demicontinuous and monotone, where $G$ is open and bounded. If $M \subset X^{*}$ is pathwise connected and such that $M \cap T(G) \neq \emptyset$ and $M \cap \overline{T(\partial G)}=\emptyset$, then $M \subset T(G)$. 
Proof. Without loss of generality, we assume that $0 \in M \cap T(G), 0 \in G$ and $T(0)=0$. In fact, if this is not already true, we fix $x_{0}^{*} \in M \cap T(G)$ and consider instead of $T$ the operator $\widetilde{T} x \equiv T\left(x+x_{0}\right)-x_{0}^{*}, x \in \overline{\widetilde{G}}$, where $T x_{0}=x_{0}^{*}$ and $\widetilde{G}=G-x_{0}$. We also consider instead of $M$ the set $\widetilde{M} \equiv M-x_{0}^{*}$. It is easy to see that $\widetilde{M}$ is pathwise connected, $\widetilde{M} \cap \overline{\widetilde{T}(\partial \widetilde{G})}=\emptyset$ and $\widetilde{M} \cap \widetilde{T}(\widetilde{G}) \ni 0,0 \in \widetilde{G}$ and $\widetilde{T}(0)=0$.

We consider the operators $T_{n} x \equiv T x+(1 / n) J x$. We fix $x^{*} \in M$ and let $s$ : $[0,1] \rightarrow M$ be continuous and such that $s(0)=0$ and $s(1)=x^{*}$. As in the proof of Guan's Theorem 1, we can see now that there is $n=n_{0}$ such that $s(t) \notin T_{n}(\partial G)$ for all $n \geq n_{0}$. From this point on we consider only such values of $n$. Since $T_{n}$ is demicontinuous, monotone, one-to-one and of type $(S)$ on each closed ball contained in $G$, Theorem A says that $T_{n}(G)$ is open. Thus, the set $Q_{n} \equiv\{t \in[0,1]: s(t) \in$ $\left.T_{n}(G)\right\}$ is open in $[0,1]$, because it equals the set $s^{-1}\left(T_{n}(G)\right)$. We also know that $Q_{n}$ is nonempty, because $0 \in Q_{n}$. Now, let us show that $Q_{n}$ is closed in $[0,1]$. Let $\left\{t_{m}\right\} \subset Q_{n}$ be such that $t_{m} \rightarrow t \in[0,1]$. We have, for some sequence $\left\{x_{m}\right\} \subset G$, $s\left(t_{m}\right)=T_{n}\left(x_{m}\right)$ and $s\left(t_{m}\right) \rightarrow s(t)$. From

$$
s\left(t_{m}\right)=T x_{m}+(1 / n) J x_{m},
$$

we get

$$
(1 / n) \limsup _{m, j \rightarrow \infty}\left\langle J x_{m}-J x_{j}, x_{m}-x_{j}\right\rangle \leq 0 .
$$

We may assume that $x_{m} \rightarrow \widetilde{x} \in X$ and $J x_{m} \rightarrow y^{*} \in X^{*}$. Applying Lemma A, we get that $x_{m} \rightarrow \widetilde{x}$. Using the demicontinuity of $T_{n}$, we obtain $s(t)=T_{n} \widetilde{x}$. Since $s(t) \notin T_{n}(\partial G)$, we have that $s(t) \in T_{n}(G)$. Thus, $Q_{n}$ is also closed in $[0,1]$. It follows that $Q_{n}=[0,1]$. This implies that there is a sequence $x_{n} \in G$ such that

$$
T x_{n}+(1 / n) J x_{n}=x^{*} .
$$

The monotonicity of $T$ yields

$$
\left\langle(1 / n) J x_{n}-(1 / m) J x_{m}, x_{n}-x_{m}\right\rangle \leq 0 .
$$

Since $\{1 / n\}$ is decreasing to 0 and $\left\{\left\|x_{n}\right\|\right\}$ is bounded, Lemma $\mathrm{C}$ implies that $x_{n} \rightarrow$ (some) $x \in \bar{G}$. By demicontinuity and (22), $T x=x^{*}$. Obviously, since $x^{*} \in M$ we cannot have $x^{*} \in T(\partial G)$. Thus, $x^{*} \in T(G)$. We conclude that $M \subset T(G)$.

Corollary 6. Let the assumptions of Proposition 2 be satisfied with $T$ also of type $(S)$ and $\overline{T(\partial G)}$ replaced by $T(\partial G)$. Then the conclusion of Proposition 2 holds.

Proof. It suffices to notice that since $T$ is of type $(S)$, the set $T(\partial G)$ is closed. Thus, again, $M \cap \overline{T(\partial G)}=\emptyset$ and the result follows from Proposition 2.

Theorem 5. Let $G \subset X$ be open and bounded, and let $T: \bar{G} \rightarrow X^{*}$ be demicontinuous and monotone. Assume that there exists $x_{0} \in G$ such that $\left\|T x_{0}\right\|<\|T x\|, x \in$ $\partial G$, with $r \equiv \inf \{\|T x\|: x \in \partial G\}>0$. Then $B_{r}(0) \subset T(G)$.

Proof. We note first that we may take $x_{0}=0$. In fact, if this is not already true, we consider instead of $T$ the operator $T_{1} x \equiv T\left(x+x_{0}\right), x \in \bar{G}_{1}$, where $G_{1}=G-x_{0}$. Thus, we have $\|T(0)\|<\|T x\|, x \in \partial G$. We first introduce a perturbation to the problem and then follow, generally, Guan's approach in [10, Theorem 5]. We observe that for every $\alpha>0$ the operator $T+\alpha J$ is a demicontinuous, monotone operator. 
It is also of type $\left(S_{q}\right)$. In fact, assume that $\left\{x_{n}\right\} \subset G$ and $x_{n} \rightarrow x,(T+\alpha J) x_{n} \rightarrow 0$, for some $\alpha>0$. We may also assume that $J x_{n} \rightarrow y^{*}$. Then the sequence of terms $u_{n} \equiv(T+\alpha J) x_{n}$ is Cauchy. This and

$$
\begin{aligned}
0 & \leq \alpha\left\langle J x_{n}-J x_{m}, x_{n}-x_{m}\right\rangle \\
& \leq\left\langle T x_{n}-T x_{m}, x_{m}-x_{n}\right\rangle+\alpha\left\langle J x_{n}-J x_{m}, x_{n}-x_{m}\right\rangle \\
& =\left\langle u_{n}-u_{n}, x_{n}-x_{m}\right\rangle \\
& \leq\left\|u_{n}-u_{m}\right\|\left\|x_{n}-x_{m}\right\|
\end{aligned}
$$

imply

$$
\lim _{m, n \rightarrow \infty}\left\langle J x_{n}-J x_{m}, x_{m}-x_{n}\right\rangle=0 .
$$

Thus, Lemma A applies here to yield $x_{n} \rightarrow x \in \bar{G}$ and $(T+\alpha J) x_{n} \rightarrow(T+\alpha J) x=0$. We are going to show that $T x+\alpha J x \neq \mu J x, x \in \partial G$, for every $\alpha>0$. To this end, we assume that this is false and let $x \in \partial G$ be such that $T x+\alpha J x=\mu J x$, or $T x=(\mu-\alpha) J x$. We have

$$
\langle T x-T(0), x\rangle+\langle T(0), x\rangle=(\mu-\alpha)\langle J x, x\rangle,
$$

which implies

$$
-\|T(0)\|\|x\| \leq(\mu-\alpha)\|J x\|\|x\|
$$

and $\|T(0)\| \geq(\alpha-\mu)\|J x\|=\|T x\|$. This is a contradiction to our assumed boundary condition. Thus, we can now apply Theorem 4 in order to conclude that the equation $T x+\alpha J x=0$ is solvable with $x=x_{\alpha} \in G$ for every $\alpha>0$. Since $G$ is bounded, we may take $\alpha \rightarrow 0$ in order to obtain that $T x_{\alpha} \rightarrow 0 \in \overline{T(G)}$. This says that for every $\epsilon \in(0, r)$ we have $\overline{B_{r-\epsilon}(0)} \cap T(G) \neq \emptyset$. Also, since $\|T x\| \geq r, x \in \partial G$, we have $\overline{B_{r-\epsilon}(0)} \cap \overline{T(\partial G)}=\emptyset$. By Proposition $2, \overline{B_{r-\epsilon}(0)} \subset T(G)$. Since $\epsilon \in(0, r)$ is arbitrary, we have $B_{r}(0) \subset T(G)$.

Theorem 5 improves the main part of Theorem 5 of Guan [10], where the set $G$ is assumed to be convex.

\section{A partial answer to Nirenberg's PRoblem}

Let $H$ be a real Hilbert space. In this section we give a partial answer to Nirenberg's problem: Let $T: H \rightarrow H$ be continuous and such that $T(0)=0$. Assume that $T$ maps a neighborhood of zero onto a neighborhood of zero and is expansive $(\|T x-T y\| \geq\|x-y\|, x, y \in H)$. Is $T$ surjective? Chang and Li showed in [7] that this is true in a Banach space $X$ if the Fréchet derivative $T^{\prime}(x)$ exists for each $x \in X$ and satisfies a weak type of continuity condition. Morel and Steinlein showed in [32] that Nirenberg's mapping is not generally surjective in $l^{1}$.

We show that if the operator $T$ satisfies a certain monotonicity type of condition, then the mapping $T$, in a real Hilbert space $H$, is surjective, without assuming that $\operatorname{int} R(T) \neq \emptyset$. The additional condition that we assume is that there exists a constant $\alpha \in(0,1)$ such that

$$
\langle T x-T y, x-y\rangle \geq-\alpha\|x-y\|^{2}, \text { for every } x, y \in H .
$$

We have the following theorem.

Theorem 6. Let $T: H \rightarrow H$ be continuous and expansive. Then $T$ is surjective if and only if one of the following statements holds true. 
(i) the mapping $U=I+T^{-1}: R(T) \rightarrow H$ is maximal monotone;

(ii) the mapping $T+\mu I$ is surjective for some (or, equivalently, all) $\mu \in(0,1)$.

In particular, $T$ is surjective if there exists a constant $\alpha \in(0,1)$ such that (23) holds.

Proof. To show (i), let us assume that the operator $U$ is maximal monotone. It is easy to see that $D(U)=R(T)$ is a closed set. By the Kirszbraun-Valentine theorem, we may extend the mapping $T^{-1}$ to a non-expansive mapping $\widetilde{T}$ defined on all of $H$. Then the mapping $\widetilde{U}=I+\widetilde{T}$ is a continuous monotone mapping defined on all of $H$. By a well-known result, such a mapping is maximal monotone. Since $\widetilde{U}$ is an extension of the mapping $U$, we have a contradiction to the maximality of $U$, unless $D(U)=R(T)=H$. Conversely, if $R(T)=H$, then the operator $U$ is maximal monotone.

To show (ii), it suffices to observe that $U$ is maximal monotone in the Hilbert space $H$ if and only if $R(U+\lambda I)=H$ for some (or, equivalently, all) $\lambda>0$. This is the same as saying that $R\left(\lambda I+T^{-1}\right)=H$ for some (or, equivalently, all) $\lambda>1$. Since the equation $\lambda y+T^{-1} y=p, y \in R(T)$, is equivalent to the equation $T x+(1 / \lambda) x=(1 / \lambda) p, x \in H$, for every $p \in H$, we have that $U$ is maximal monotone if and only if the operator $T+(1 / \lambda) I$ is surjective for some (or, equivalently, all) $\lambda>1$. This completes the proof of case (ii).

Now, let (23) hold for some $\alpha \in(0,1)$ and fix $\epsilon \in(0,1-\alpha)$. Then, for every $x, y \in H$, we have

$$
\langle T x-T y+(\alpha+\epsilon)(x-y), x-y\rangle \geq \epsilon\|x-y\|^{2} .
$$

Thus, $T+(\alpha+\epsilon) I$ is now a continuous, strongly monotone operator defined on all of $H$. By well-known results, it is maximal monotone and surjective. By Part (ii), with $\mu=\alpha+\epsilon<1, T$ is surjective.

\section{Discussion. Example}

It is easy to see that all the zeros of a maximal monotone operator are obtained in the way suggested by Theorem 3 . In fact, if $x_{0} \in T^{-1}(0)$, then

$$
(t T+J)^{-1} J x_{0}=x_{0} \text { and } J_{t} x_{0}=x_{0},
$$

i.e., the limits

$$
\lim _{t \rightarrow \infty}(t T+J)^{-1} x^{*} \text { and } \lim _{t \rightarrow \infty} J_{t} x
$$

are common fixed points of the mappings $(t T+J)^{-1} J x$ and $J_{t} x$, respectively.

Some results from Section 3 can be extended to classes of regular operators. Let us call an operator $T: X \supset D(T) \rightarrow 2^{X^{*}}$ "regular at $p^{*} \in X^{*}$ " if the inclusion $T x+\lambda J x \ni p^{*}$ has a solution $x=x_{\lambda}$ for each $\lambda>0$. Naturally, certain boundedness properties of the (generally multi-valued) resolvents of regular operators along with some closedness properties (like generalized pseudo-monotonicity (cf. [35, pp. 148150]) should provide some range results for such operators.

The solutions of the stabilizing equation (18) are bounded for $t \in R_{+}$without the assumption that $G$ is a bounded set. This fact might be of some help in future considerations. In order to show it, assume that $G$ is unbounded, let

$$
Q=\left\{x \in G: g_{t}(x)=0, \text { for some } t \in R_{+}\right\}
$$


and let $x_{t} \in Q$, for some $t \in R_{+}$. If $t=0$, then $x_{t}=x_{0}$. Let $t>0$. Then

$$
t\left\langle T x_{t}-T x_{0}, x_{t}-x_{0}\right\rangle+t\left\langle T x_{0}, x_{t}-x_{0}\right\rangle+\alpha t\left(\left\|x_{t}\right\|-\left\|x_{0}\right\|\right)^{2} \leq 0,
$$

or

$$
\alpha t\left(\left\|x_{t}\right\|-\left\|x_{0}\right\|\right)^{2} \leq t\left|\left\langle T x_{0}, x_{t}-x_{0}\right\rangle\right| \leq t\left\|T x_{0}\right\|\left\|x_{t}-x_{0}\right\|,
$$

and, finally,

$$
\alpha\left(\left\|x_{t}\right\|-\left\|x_{0}\right\|\right)^{2} \leq\left\|T x_{0}\right\|\left\|x_{t}-x_{0}\right\|,
$$

which shows the boundedness of the set $Q$. Naturally, this remark raises the question as to whether Theorem 4 is actually true without the assumption that $G$ is a bounded set.

The invariance of domain result of Park [34], which is Theorem A with "locally of type $(S)$ " replaced by "of type $(S)$ ", is an easy consequence of Guan's Theorem 1 in [10]. In fact, this is demonstrated in Lemma 5 of [12]. An invariance of domain result was given there, independently, for open, bounded and convex sets, but the general case of open sets, which is Theorem A, follows trivially from that result. For the solution of this problem with $T$ continuous, but not necessarily monotone, the reader is referred to the book of Skrypnik [38, p. 59, Theorem 7.4].

Open Problem 1. Let $T: X \supset \bar{G} \rightarrow 2^{X^{*}}\left(T: X \supset \bar{G} \rightarrow 2^{X}\right)$ be monotone (accretive) and injective (i.e., $u \in T x, v \in T y$ and $u=v$ imply that $x=y$ ), where $G$ is open and bounded. Under what further continuity/closedness assumptions can we conclude that $T G$ is an open set?

Naturally, the assumptions on the space $X$, made for the monotone case above, are not necessarily needed for the accretive case. Some kind of upper semicontinuity assumption on the operator $T$ above is preferable to its continuity w.r.t. the Hausdorff metric.

It should be noted that if, for example, $T$ is as in Open Problem 1 and strongly monotone, then the openness of $T G$ would imply the openness of $T G_{1}$, for every open set $G_{1} \subset G$. In fact, in this case we have that $T^{-1}$ is well-defined and continuous on the open set $T G \subset X^{*}$. This implies that the multi-valued inverse image operator of $T^{-1}$, i.e., the operator $T$ itself, maps relatively open sets of its domain onto relatively open subsets of its range. This says that $T G_{1}$ is open in $T G$. However, the openness of $T G$ in $X^{*}$ implies that $T G_{1}$ is also open in $X^{*}$. Since $T$ is strongly monotone, we have that $T \bar{G}_{1}$ is closed. Such operators $T$ are important for the development of the degree theoretic solvability of problems of the type

$$
T x+C x \ni 0,
$$

where $C: \bar{G} \rightarrow X^{*}$ is a compact operator. Other related problems involve the existence of eigenvalues $\lambda$ for

$$
T x+\lambda C x \ni 0
$$

or "ranges of sums" of the types

$$
\operatorname{int}(R(T)+R(C))=\operatorname{int} R(T+C), \quad \operatorname{int} R(T) \subset R(T+C),
$$

and

$$
\operatorname{int} R(C) \subset R(T+C)
$$


By an argument like the above, we may also conclude that if $T: X \supset D(T) \rightarrow 2^{X^{*}}$ is maximal monotone and strongly monotone, then, for every open set $G \subset X$ we have that $T(D(T) \cap G)$ is open in $X^{*}$ and $T(D(T) \cap \bar{G})$ is closed in $X^{*}$. For such considerations, the reader is referred to the papers [8]-[9], [25] and [26].

Open Problem 2. Let $T: X \supset D(T) \rightarrow 2^{X^{*}}\left(T: X \supset D(T) \rightarrow 2^{X}\right)$ be maximal monotone (m-accretive) and $C: \overline{D(T)} \rightarrow X^{*}(C: X \supset \overline{D(T)} \rightarrow X)$ completely continuous (with $X^{*}$ uniformly convex). Establish necessary and sufficient conditions for the existence of a zero of the operator $T+C$.

As far as the author knows, no such conditions are known for nontrivial nonlinear operators $T$ and $C$. The papers [37] and [24] contain useful information in this direction.

Open Problem 3. Let $X^{*}$ be uniformly convex and let $G$ be an open, bounded subset of $X$. Develop a degree theory, in the sense of Browder, for the sum of two operators $T+C$, where $T: X \supset \bar{G} \rightarrow X$ is demicontinuous and strongly accretive, and $C: \bar{G} \rightarrow X$ is compact.

The degree theory referred to above should be developed independently of the degree theories involving $A$-proper mappings in Banach spaces containing schemes of approximation-solvability. It would be interesting to see such a degree theory based on the Leray-Schauder degree $d_{L S}\left(I-C T^{-1}, T G, 0\right)$. This type of approach would involve the investigation of basic homotopy properties of the degree on timedependent sets. This is the approach of Browder [5]. However, Browder studied in [5] continuous operators $T$ which were local homeomorphisms. The invariance of domain result of the author used in [19] would be useful in solving Problem 3. For various other degree theories involving accretive operators, we cite the short survey paper of the author [18].

As we mentioned earlier, Skrypnik developed in [38] a degree theory for bounded demicontinuous operators satisfying condition $\left(S_{+}\right)$. The book [38] contains a good number of examples involving such operators. These examples, suitably modified, may be applied to the theory herein.

Example 1. For the purpose of illustrating the applicability of some of our results, we elaborate below on an example involving a nonlinear partial differential operator. Let $D$ be a bounded open subset of $\mathcal{R}^{N}, N \geq 1$. Let $p \in[2, \infty)$ and $p^{-1}+q^{-1}=1$. Let $x \in \mathcal{R}^{N}$ be the vector $x=\left(\xi_{1}, \xi_{2}, \ldots, \xi_{N}\right)$ and $D_{i}=\partial / \partial \xi_{i}$. Let $X=W_{0}^{1, p}$ and consider the boundary value problem

$$
\begin{aligned}
& -\sum_{i=1}^{N} D_{i}\left(\left|D_{i} u(x)\right|^{p-2} D_{i} u(x)\right)=f, \quad x \in D, \\
& u(x)=0, \quad x \in \partial G,
\end{aligned}
$$

where $f \in L^{q}(D)$ is given. The problem (24) has a corresponding generalized problem given by

$$
a(u, v)=b(v), \quad v \in X,
$$


where

$$
\begin{aligned}
a(u, v) & =\int_{D}\left(\sum_{i=1}^{N}\left|D_{i} u\right|^{p-2} D_{i} u D_{i} v\right) d x, \\
b(v) & =\int_{D} f v d x .
\end{aligned}
$$

By the equation (25) we mean the following: given $f \in L^{q}(D)$, find $u \in X$ such that (25) is satisfied. From Zeidler [40, p. 568] we know that (25) is equivalent to the equation

$$
T u=b,
$$

where $T: X \rightarrow X^{*}$ is defined by $\langle T u, v\rangle=a(u, v), u, v \in X$. The operator $T$ is continuous, monotone, coercive and bounded. Consequently, Proposition 2 and Theorem 5 are directly applicable to this operator $T$. In particular, from the coercivity of $T$ [40, p. 569] we obtain

$$
\|T u\| \geq 2^{2-p}\|u\|^{p-1}, \quad u \in X,
$$

where the constant $c=2^{2-p}$ comes from the inequality

$$
\left(|x|^{p-2} x-|y|^{p-2} y\right)(x-y) \geq c|x-y|^{p}, \quad x, y \in \mathcal{R}
$$

(cf. [28] for this inequality in a real Hilbert space with best possible constant $c$ ). It follows that, for an open set $G \subset X$ containing zero, we have

$$
\inf \{\|T u\|: u \in \partial G\} \geq c r^{p-1},
$$

where $r>0$ is defined by

$$
r=\inf \{\|u\|: u \in \partial G\} .
$$

Since $T(0)=0$, we see that Theorem 5 provides us with the qualitative information that for every open set $G \subset X$, with its boundary points having norms bounded below by $r$, the open ball of $X^{*}$ around zero with radius $c r^{p-1}$ lies in $T G$. It is essential to mention here that this conclusion, for a fixed $r>0$, remains true if $T$ is additively perturbed by any demicontinuous monotone operator defined only on the set $\bar{G}$ and taking the value 0 at $x=0$. Given any open and bounded set $G \subset X$, any pathwise connected set $M \subset X^{*}$ lies in the set $T(G)$ if $0 \in M$ and $M$ does not intersect $T(\partial G)$.

On the other hand, since $0 \in X$ is the only zero of the operator $T$, we have $T^{-1}(0)=0$, and Theorem 3 provides us with the asymptotic behavior

$$
\lim _{t \rightarrow \infty}(t T+J)^{-1} x^{*}=0 \quad \text { and } \quad \lim _{t \rightarrow \infty}\left(I+t J^{-1} T\right)^{-1} x=0
$$

for every $x \in X$ and every $x^{*} \in X^{*}$. The same asymptotic behavior holds for possibly discontinuous maximal monotone operators as in the examples of [26].

It would be interesting to see a theorem like Theorem 5 , but with operators $T$ which do not have a monotonicity property. In this connection we mention Skrypnik's Theorem 5.1 in [38], where an analogous statement has been obtained for an operator $T: \overline{B_{r}(0)} \rightarrow X^{*}$. In that theorem $T$ is bounded, demicontinuous, odd on $\partial B_{r}(0)$, and satisfies the well-known condition $(S)_{+}$. The space $X$ is assumed to be separable. Skrypnik's conclusion is that the condition

$$
\left\|h^{*}\right\|<\|T x\|, \quad x \in \partial B_{r}(0),
$$

for some $h^{*} \in X^{*}$, implies $h^{*} \in T\left(B_{r}(0)\right)$. 
For the continuous case of the above result we cite Theorem 17.4 on p. 278 of Browder's book [5].

There have been some developments concerning Problems 1-3 since this paper was submitted. In connection with Problems 1 and 2, the reader is referred to the author's paper "An invariance of domain result for multi-valued maximal monotone operators whose domains do not necessarily contain any open sets", Proc. Amer. Math. Soc. 125 (1997), 1469-1478 (MR 97g:47053).

An answer to Problem 3 has been given in the paper by the author and J. Lin, "Homotopy invariance of parameter-dependent domains and perturbation theory for maximal monotone and $m$-accretive operators in Banach spaces", to appear.

\section{REFERENCES}

1. M. Altman, A fixed point theorem in Hilbert space, Bull. Acad. Polon. Sci. Cl. III 5 (1957), 89-92. MR 19:297b

2. M. Altman, A fixed point theorem in Banach space, Bull. Acad. Polon. Sci. Cl. III 5 (1957), 17-22. MR 19:297a

3. V. Barbu, Nonlinear semigroups and differential equations in Banach spaces, Noordhoff, Leyden, 1975. MR 52:11166

4. H. Brézis, M. G. Crandall and A. Pazy, Perturbations of nonlinear maximal monotone sets in Banach space, Comm. Pure Appl Math. 23 (1970), 123-144. MR 41:2454

5. F. Browder, Nonlinear operators and nonlinear equations of evolution in Banach spaces, Proc. Symp. Pure Appl. Math., 18, Part 2, Providence, 1976. MR 53:8982

6. F. Browder, The degree of mapping and its generalizations, Contemp. Math. 21 (1983), 15-40. MR 85e: 47086

7. K. C. Chang and S. Li, A remark on expanding maps, Proc. Amer. Math. Soc. 85 (1982), 583-585. MR 83j: 47045

8. Z. Ding and A. G. Kartsatos, Nonzero solutions of nonlinear equations involving compact perturbations of accretive operators in Banach spaces, Nonlinear Anal. TMA 25 (1995), 13331342. MR 97d: 47072

9. Z. Ding and A. G. Kartsatos, p-regular mappings and alternative results for perturbations of m-accretive operators in Banach spaces, Topol. Meth. Nonlinear Anal. 5 (1995), 291-304. MR 96k:47107

10. Z. Guan, Ranges of operators of monotone type in Banach spaces, J. Math. Anal. Appl. 174 (1993), 256-264. MR 95b:47086

11. Z. Guan, Solvability of semilinear equations with compact perturbations of operators of monotone type, Proc. Amer. Math. Soc. 121 (1994), 93-102. MR 94g:47080

12. Z. Guan and A. G. Kartsatos, Solvability of nonlinear equations with coercivity generated by compact perturbations of m-accretive operators in Banach spaces, Houston J. Math. 21 (1995), 149-188. MR 96c:47099

13. Z. Guan and A. G. Kartsatos, On the eigenvalue problem for perturbations of nonlinear accretive and monotone operators in Banach spaces, Nonlinear Anal. TMA 27 (1996), 125141. MR 97b: 47053

14. Z. Guan and A. G. Kartsatos, Ranges of perturbed maximal monotone and m-accretive operators in Banach spaces, Trans. Amer. Math. Soc. 347 (1995), 2403-2435. MR 95i:47096

15. N. Hirano and A. K. Kalinde, On perturbations of $m$-accretive operators in Banach spaces, Proc. Amer. Math. Soc. 124 (1996), 1183-1190. MR 96g:47058

16. D. R. Kaplan and A. G. Kartsatos, Ranges of sums and control of nonlinear evolutions with preassigned responses, J. Optim. Theory Appl. 81 (1994), 121-141. MR 96a:93008

17. A. G. Kartsatos, Some mapping theorems for accretive operators in Banach spaces, J. Math. Anal. Appl 82 (1981), 169-183. MR 82j:47068

18. A. G. Kartsatos, Recent results involving compact perturbations and compact resolvents of accretive operators in Banach spaces, Proceedings of the First World Congress of Nonlinear Analysts, Tampa, Florida, 1992, vol. III, Walter De Gruyter, New York, 1995, pp. 2197-2222. MR 97a:00029 
19. A. G. Kartsatos, On compact perturbations and compact resolvents of nonlinear m-accretive operators in Banach spaces, Proc. Amer. Math. Soc. 119 (1993), 1189-1199. MR 94c:47076

20. A. G. Kartsatos, Sets in the ranges of sums for perturbations of nonlinear m-accretive operators in Banach spaces, Proc. Amer. Math. Soc. 123 (1995), 145-156. MR 95c:47072

21. A. G. Kartsatos, On the construction of methods of lines for functional evolutions in general Banach spaces, Nonlinear Anal. TMA 25 (1995), 1321-1331. MR 96h:34164

22. A. G. Kartsatos, A compact evolution operator generated by a time-dependent m-accretive operator in a general Banach space, Math. Ann. 302 (1995), 473-487. MR 96c:47104

23. A. G. Kartsatos, Sets in the ranges of nonlinear accretive operators in Banach spaces, Studia Math. 114 (1995), 261-273. MR 97h:47053

24. A. G. Kartsatos, Degree theoretic solvability of inclusions involving perturbations of nonlinear m-accretive operators in Banach spaces, Yokohama Math. J. 42 (1994), 171-182. MR 96c: 47098

25. A. G. Kartsatos, On the perturbation theory of $m$-accretive operators in Banach spaces, Proc. Amer. Math. Soc. 124 (1996), 1811-1820. MR 96h:47070

26. A. G. Kartsatos, New results in the perturbation theory of maximal monotone and $m$-accretive operators in Banach spaces, Trans. Amer. Math. Soc. 348 (1996), 1663-1707. MR 96j:47050

27. A. G. Kartsatos and X. Liu, Nonlinear equations involving compact perturbations of $m$ accretive operators in Banach spaces, Nonlinear Anal. TMA 24 (1995), 469-492. MR 96c: 47079

28. A. G. Kartsatos and R. D. Mabry, Controlling the space with pre-assigned responses, J. Optim. Theory Appl. 54 (1987), 517-540. MR 88j:49024

29. W. A. Kirk and R. Schöneberg, Zeros of $m$-accretive operators in Banach spaces, Israel J. Math. 35 (1980), 1-8. MR 82a:47049

30. C. Morales, Nonlinear equations involving m-accretive operators, J. Math. Anal. Appl. 97 (1983), 329-336. MR 85d:47055

31. C. Morales, Existence theorems for demicontinuous accretive operators in Banach spaces, Houston J. Math. 10 (1984), 535-543. MR 86d:47063

32. J. M. Morel and H. Steinlein, On a problem of Nirenberg concerning expanding maps, J. Funct. Anal. 59 (1984), 145-150. MR 86b:47116

33. L. Nirenberg, Topics in nonlinear functional analysis, Lecture Notes, Courant Inst., New York Univ., New York, 1974. MR 58:7672

34. J. A. Park, Invariance of domain theorem for demicontinuous mappings of type ( $\left.S_{+}\right)$, Bull. Korean Math. Soc. 29 (1992), 81-87. MR 93d:47134

35. D. Pascali and S. Sburlan, Nonlinear mappings of monotone type, Sijthoff and Noordhoff Intern. Publ., Bucureşti, Romania, and Sijthoff \& Noordhoff, Alphen aan den Rijn, 1978. MR 80g: 47056

36. S. Reich, Strong convergence theorems for resolvents of accretive operators in Banach spaces, J. Math. Anal. Appl. 75 (1980), 287-292. MR 82a:47050

37. S. Reich and R. Torrejón, Zeros of accretive operators, Comment. Math. Univ. Carolinae 21 (1980), 619-625. MR 81k:47075

38. I. V. Skrypnik, Methods for analysis of nonlinear elliptic boundary value problems, Transl. Math. Monographs, 139, A.M.S., Providence, 1994. MR 95i:35109

39. G. H. Yang, The ranges of nonlinear mappings of monotone type, J. Math. Anal. Appl. 173 (1993), 165-172. MR 94b:47069

40. E. Zeidler, Nonlinear Functional Analysis and its Applications, II/B, Springer-Verlag, New York, 1990. MR 91b:47002

Department of Mathematics, University of South Florida, Tampa, Florida 33620-5700

E-mail address: hermes@tarski.math.usf .edu 